Gazi University
Journal of Science
http://dergipark.gov.tr/gujs

\title{
Reliability Estimation in Multicomponent Stress-Strength for Generalized Inverted Exponential Distribution Based on Ranked Set Sampling
}

\author{
Amal HASSAN ${ }^{10}$, Heba NAGY* \\ 5 Dr. Ahmed Zoweil St., Dokki, Faculty of Graduate Studies for Statistical Research (Cairo University, Department of Mathematical Statistics), \\ 12613, Giza, Egypt
}

\section{Highlights}

- This paper focuses on reliability estimation in a multicomponent stress-strength model.

- Three sampling schemes are employed for estimation problem.

- A simulation study is provided for illustrative purposes.

\section{Article Info}

Received:30 June 2020

Accepted:24 Feb 2021

Keywords

Generalized inverted exponential

Multicomponent model

Ranked set sampling

Reliability estimation

\begin{abstract}
Stress-strength models are considered of great significance due to their applicability in varied fields. We address the estimation of the system reliability of a multicomponent stress-strength model, say $R_{s, k}$, of an $s$ out of $k$ system when the pair stress and strengths are drawn from a generalized inverted exponential distribution. The system is deemed as working if at least $s$ out of $k$ strengths be more than its stress. We obtain the reliability estimators when the data of strength and stress distributions are collected from three sampling schemes, specifically; simple random sampling, ranked set sampling, and median ranked set sampling. We obtain four estimators of $R_{s, k}$ out from median ranked set sampling. The behavior of different estimates is examined via a simulation study based on mean squared errors and efficiencies. The simulation studies point out that the reliability estimates of $R_{s, k}$, from the ranked set sampling scheme are preferred than other estimates picked from the simple random sample and median ranked set sampling in a majority of the situations. The theoretical studies are explained with the aid of real data analysis.
\end{abstract}

\section{INTRODUCTION}

In many circumstances where the exact measurements of the characteristic under study are costly or hard to get, but sorting them (in small sets) is cheap or easy, ranked set sampling (RSS) scheme is indeed suitable than simple random sampling (SRS). The RSS is another cost-effective sampling method that can be utilized for a given sampling unit of an experiment or a study. The RSS was originally prepared in [1] for estimating the mean of grass yield. He observed that whereas getting the exact value of yield of a plot is hard and takes a long time, one can easily rank closely plots in terms of their pasture yield by an eye examination.

The RSS design is formed as: Randomly take random samples of size $n$ from the population study each of size $n$, then rank the units in each sample according to the interested variable by optical inspection or by any other economical process. Then the lowest and second lowest units from the first and second samples are chosen for substantial measurement. Repeat this procedure up-till the highest unit from the $n^{\text {th }}$ sample is chosen for measurements. Thus all of $n$ measured units, which exemplified one cycle, are collected. This procedure may be worked out $r$ times until the number of $n r$ units is yielded.

Reference [2] offered the mathematical theory, which supports McIntyre's claims. [3] indicated that the mean of RSS is unbiased and more efficient than the mean of SRS. [4] provided some tests of exponentiated Pareto distribution in case of extreme RSS. [5] handled with the maximum likelihood (ML) and Bayesian 
estimators using RSS. Modified tests of the Weibull model were provided in [6]. For some more knowledge about RSS, see [7-9].

Various researchers proposed a modification of the RSS design to get better estimators for the population mean. Median RSS (MRSS) is perhaps one of the popular schemes (see [10]). MRSS strategy is formed as: we select $n$ random samples randomly from the target population each of size $n$. The units within each sample are sorted according to the interesting variable. For an odd sample size, choose the median member of each arranged set. For an even sample size, take from the first $n / 2$ samples the $(n / 2)^{\text {th }}$ smallest sorted member and from the second $n / 2$ samples the $((n / 2)+1)^{\text {th }}$ smallest ordered member. To get more samples from MRSS, the cycle is worked out $r$ steps to gain a sample of size $n r$ members.

In the area of mechanical engineering stress-strength (SS) reliability models are frequently serviced to characterize the life of a unit that possesses a random strength $X$ and is submitted to a random stress $Y$. The SS reliability, mathematically is formed as $R=P(Y<X)$, that is, the system will stop working if the stress applied to it overrides its strength. This model was first established in [11] and expanded in [12]. Broadly, making inferences about SS reliability have been discussed extensively in the statistical literature based on SRS data by many researchers. For instance, [13] discussed the reliability estimator for the exponential distributions. [14] considered three estimators of the SS model when $X$ and $Y$ are independent exponential models. [15] considered the estimation of the SS reliability for the generalized inverted exponential (GIE) distribution from RSS. The SS reliability was discussed for independent Burr type XII distribution under some types of RSS [16, 17]. Estimation of the SS model for Weibull distribution was provided in [18]. Reliability estimation of the SS model was considered in [19] for two independent Lindley populations. [20] addressed the reliability estimation for $X$ and $Y$ are independent exponentiated Pareto populations using RSS.

A multicomponent structure consists of $k$ strength members (ingredients), where $k$ identically independent distributed (iid) random variables $X_{1}, X_{2}, \ldots, X_{k}$ and each member (ingredient) faces a random stress $Y$. The structure is considered as active only if at least $s$ out of $k(s<k)$ strengths override the stress. Consider $X_{1}$, $X_{2}, \ldots, X_{k}$ be iid with common cumulative distribution function (CDF) $F(x)$. Also, let $G(y)$ be the CDF of a random stress $Y$. The reliability in the multicomponent SS (MSS) model adopted in [21] is assigned by:

$$
\begin{aligned}
R_{s, k} & =P\left[\text { at least } s \text { of the }\left(X_{1}, X_{2}, \ldots, X_{k}\right) \text { exceed } Y\right] \\
& =\sum_{i=s}^{k}\left(\begin{array}{l}
k \\
i
\end{array}\right) \int_{-\infty}^{\infty} F(y)^{k-i}[1-F(y)]^{i} d G(x) .
\end{aligned}
$$

Systems of MSS type can be found in industrial and military applications (see [22]). Estimation of the MSS reliability was treated and applied in some areas by several authors. For example [23] handled the reliability of the MSS when both $X$ and $Y$ variables are distributed as generalized Pareto. [24, 25] discussed the reliability of the MSS for exponentiated Pareto distribution. [26] considered the MSS reliability estimator for generalized exponential distribution. [27] estimated the MSS reliability when $X$ and $Y$ are independent Burr XII distribution. [28] discussed the estimation of the MSS reliability when $X$ and $Y$ are independent Burr XII distribution under selective RSS. [29] discussed $s$-out-of- $k$ reliability estimator for Weibull distribution from record values.

Applications of the RSS scheme are not limited to the agricultural field, but also include forestry, medicine environmental monitoring and entomology. In the literature, there are few studies that had been performed about the SS problem incorporating multicomponent systems based on the RSS technique. Therefore, our objective here is to assess the MSS reliability estimators for GIE distribution depend on SRS, RSS and MRSS. We attain the simulation study to compare estimates of the suggested sampling schemes. This article is outlined as follows. Section 2 displays the model description and the reliability of the structure. Section 3 assigns to the ML estimator of $R_{\mathrm{s}, \mathrm{k}}$ under SRS. Section 4 deals with the MSS reliability estimator under RSS. Section 5 presents different MSS reliability estimates when the available observations of $X$ and $Y$ 
variables are gathered from MRSS for even or odd sample sizes. Simulation studies, as well as applications to real data, are managed, respectively in Sections 6 and 7. We conclude the paper at the end of the paper.

\section{MODEL SPECIFICATION AND THE RELIABILITY OF THE STRUCTURE}

The exponential distribution is a very simple and the most popular studied distribution in life testing. Several generalizations of the exponential distribution were proposed due to the lack of ability to model real-life phenomena with non-constant failure rates. The inverted exponential (IE) carries the inverted bathtub hazard rate which has many applications in various areas. [30] proposed the two-parameter generalization of the IE distribution known as the GIE. They have recommended that the GIE model is the acceptable model to real data compared to the IE model. The GIE model is practically employed in multiple field areas. The GIE model has a probability density function assigned as:

$$
f(x ; \alpha, \lambda)=\lambda \alpha x^{-2} \mathrm{e}^{-\lambda / x}\left(1-\mathrm{e}^{-\lambda / x}\right)^{\alpha-1} ; \quad x>0,
$$

where, $\alpha, \beta>0$ are the shape and scale parameters respectively. The CDF associated with (2) is:

$$
F(x ; \alpha, \lambda)=1-\left(1-e^{-\lambda / x}\right)^{\alpha}, x>0 .
$$

Studies about the GIE distribution were discussed by several researchers (for instance see [31-35]). Note that; for $\alpha=1$, the CDF (Equation (3)) reduces to the IE distribution.

Suppose that $X_{1}, X_{2}, \ldots, X_{k}$ be the strengths components (ingredients) of a structure that is exposed to the stress $Y$. Let $X_{1}, X_{2}, \ldots, X_{k}$ is a random sample from $\operatorname{GIE}(\lambda, \alpha)$ distribution and $Y$ is a random variable from $\operatorname{GIE}(\lambda, \beta)$ distribution are independent. The reliability of MSS for the GIE distribution can be obtained using (1) - (3) as

$$
\begin{aligned}
R_{s, k} & =\sum_{i=s}^{k}\left(\begin{array}{c}
k \\
i
\end{array}\right) \int_{0}^{\infty} \beta \lambda y^{-2} e^{-(\lambda / y)}\left\{1-\left(1-e^{-(\lambda / y)}\right)^{\alpha}\right\}^{k-i}\left\{1-e^{-(\lambda / y)}\right\}^{\alpha i+\beta-1} d y \\
& =\delta \sum_{i=S}^{K}\left(\begin{array}{c}
k \\
i
\end{array}\right) \int_{0}^{1} z^{i+\delta-1}[1-z]^{k-i} d z=\delta \sum_{i=s}^{k}\left(\begin{array}{c}
k \\
i
\end{array}\right) \mathrm{B}[i+\delta, k+1-i],
\end{aligned}
$$

such that $\delta=\frac{\beta}{\alpha}$, and $\mathrm{B}(. .$,$) is the beta function.$

\section{MSS RELIABILITY ESTIMATOR BASED ON SRS}

Here, we derive the ML reliability estimator of the MSS given the samples. We consider that $X_{1}, X_{2}, \ldots, X_{n}$ and $Y_{1}, Y_{2}, \ldots, Y_{m}$ are independent from $\operatorname{GIE}(\lambda, \alpha)$ and $\operatorname{GIE}(\lambda, \beta)$ respectively. We must obtain the ML estimators of $\alpha$ and $\beta$, to get the ML estimator of MSS reliability. The joint log-likelihood function of the observed sample is:

$$
\begin{aligned}
L & =n \ln \alpha+m \ln \beta+(m+n) \ln \lambda-2\left[\sum_{i=1}^{n} \ln x_{i}+\sum_{j=1}^{m} \ln y_{j}\right]-\lambda\left[\sum_{i=1}^{n} \frac{1}{x_{i}}+\sum_{j=1}^{m} \frac{1}{y_{j}}\right]+(\alpha-1) \sum_{i=1}^{n} \ln \left(1-e^{-\lambda / x_{i}}\right) \\
& +(\beta-1) \sum_{j=1}^{m} \ln \left(1-e^{-\lambda / y_{j}}\right) .
\end{aligned}
$$

Differentiating Equation (5) related to the population parameters, the following equations are obtained

$$
\partial \ln L / \partial \alpha=\frac{n}{\alpha}+\sum_{i=1}^{n} \ln \left(1-e^{-\left(\lambda / x_{i}\right)}\right)
$$


$\partial \ln L / \partial \beta=\frac{m}{\beta}+\sum_{j=1}^{m} \ln \left(1-e^{-\left(\lambda / y_{j}\right)}\right)$

and,

$$
\partial \ln L / \partial \ln \lambda=\frac{(m+n)}{\lambda}-\left[\sum_{i=1}^{n} \frac{1}{x_{i}}+\sum_{j=1}^{m} \frac{1}{y_{j}}\right]+\sum_{i=1}^{n} \frac{(\alpha-1)\left(x_{i}\right)^{-1}}{\left(e^{\left(\lambda / x_{i}\right)}-1\right)}+\sum_{j=1}^{m} \frac{(\beta-1)\left(y_{j}\right)^{-1}}{\left(e^{\left(\lambda / y_{j}\right)}-1\right)} .
$$

The ML estimators are derived after putting Equations (6) - (8) with zero. As seen, the ML estimator of $\alpha$ and $\beta$ is obtained as a function of $\lambda$ by using Equation (6) and Equation (7) as follows:

$$
\hat{\alpha}(\lambda)=-n\left[\sum_{i=1}^{n} \ln \left(1-e^{-\lambda / x_{i}}\right)\right]^{-1}, \quad \text { and }, \quad \hat{\beta}(\lambda)=-m\left[\sum_{j=1}^{m} \ln \left(1-e^{-\lambda / y_{j}}\right)\right]^{-1} .
$$

The ML estimators of $\alpha$ and $\beta$ can be obtained from Equation (9) when $\lambda$ is known. In the case of all unknown parameters, the parameter $\lambda$ is estimated by solving the following nonlinear equation:

$$
\omega(\lambda)=\frac{(n+m)}{\left[\sum_{i=1}^{n} \frac{1}{x_{i}}+\sum_{j=1}^{m} \frac{1}{y_{j}}\right]+\left(\frac{n}{\sum_{i=1}^{n} \ln \left(1-e^{-\left(\lambda / x_{i}\right)}\right)}+1\right)\left(\sum_{i=1}^{n} \frac{\left(x_{i}\right)^{-1}}{\left(e^{\left(\lambda / x_{i}\right)}-1\right)}\right)+\left(\frac{m}{\sum_{j=1}^{m} \ln \left(1-e^{-\left(\lambda / y_{j}\right)}\right)}+1\right)\left(\sum_{j=1}^{m} \frac{\left(y_{j}\right)^{-1}}{\left(e^{\left(\lambda / y_{j}\right)}-1\right)}\right)} .
$$

Note that $\hat{\lambda}$ is a fixed point solution of Equation (10), hence we apply iterative methodology to get the solution. Consequently, the ML estimator of the MSS reliability $R_{s . k}$ is attained after substituting the ML of $\alpha$ and $\beta$ in Equation (4).

\section{MSS RELIABILITY ESTIMATOR BASED ON RSS}

Here, the ML reliability estimator in an MSS model $R_{s, k}$ is obtained when $X$ and $Y$ are independent GIE distribution from RSS. Suppose $\left\{X_{i(i) c}, i=1,2 \ldots, m_{x} ; c=1,2, \ldots r_{x}\right\}$, where $m_{x}$ is the set size and $r_{x}$ is the number of cycles, is observed RSS with sample size $n=m_{x} r_{x}$, chosen from the GIE $(\lambda, \alpha)$. Similarly, let \{ $\left.Y_{j(j) d}, j=1,2 \ldots, m_{y} ; d=1,2, \ldots r_{y}\right\}$ is an observed RSS having sample size $m=m_{y} r_{y}$ where $m_{y}$ is the set size, accepted from the GIE $(\lambda, \beta)$. The likelihood function $L_{1}$ for the accessible samples is

$$
\begin{aligned}
L_{1}= & \prod_{c=1}^{r_{x}} \prod_{i=1}^{m_{x}} \frac{m_{x} ! \lambda \alpha}{(i-1) !\left(m_{x}-i\right) !\left(x_{i(i) c}^{2}\right)} e^{-\left(\lambda / x_{i(i) c}\right)}\left(H_{i(i) c}^{\alpha\left(m_{x}-i+1\right)-1}\right)\left\{1-H_{i(i) c}^{\alpha}\right\}^{i-1} \\
& \prod_{d=1}^{r_{y}} \prod_{j=1}^{m_{y}} \frac{\lambda\left(m_{y} !\right) \beta\left(y_{j(j) d}^{-2}\right)}{(j-1) !\left(m_{y}-j\right) !} e^{-\left(\lambda / y_{j(j) d}\right)}\left(O_{j(j) d}^{\beta\left(m_{y}-j+1\right)-1}\right)\left\{1-O_{j(j) d}^{\beta}\right\}^{j-1}
\end{aligned}
$$

where, $H_{i(i) c}=\left\langle 1-e^{-\left(\lambda / x_{i(i) c}\right)}\right\rangle$, and $O_{j(j) d}=\left\langle 1-e^{-\left(\lambda / y_{j(j) d}\right)}\right\rangle$. The log-likelihood function $L_{1}$ of $\alpha, \beta$ and $\lambda$ is written as 


$$
\begin{aligned}
\ln L_{1} & \propto r_{x} m_{x} \ln (\lambda \alpha)-2 \sum_{c=1}^{r_{x}} \sum_{i=1}^{m_{x}} \ln x_{i(i) c}-\sum_{c=1}^{r_{x}} \sum_{i=1}^{m_{x}} \frac{\lambda}{x_{i(i) c}}+\sum_{c=1}^{r_{x}} \sum_{i=1}^{m_{x}}\left[\alpha\left(m_{x}+1-i\right)-1\right] \ln H_{i(i) c} \\
& +\sum_{c=1}^{r_{x}} \sum_{i=1}^{m_{x}}(i-1) \ln \left(1-H_{i(i) c}^{\alpha}\right)+r_{y} m_{y} \ln (\lambda \beta)-2 \sum_{d=1}^{r_{y}} \sum_{j=1}^{m_{y}} \ln y_{j(j) d}-\sum_{d=1}^{r_{y}} \sum_{j=1}^{m_{y}} \frac{\lambda}{y_{j(j) d}} \\
& +\sum_{d=1}^{r_{y}}\left\langle\sum_{j=1}^{m_{y}}\left\{\left[\beta\left(m_{y}+1-j\right)-1\right] \ln O_{j(j) d}+(j-1) \ln \left(1-O_{j(j) d}^{\beta}\right)\right\}\right\rangle .
\end{aligned}
$$

The ML estimators of parameters are obtained by maximizing $\ln L_{1}$ as follows:

$$
\begin{aligned}
& \frac{\partial \ln L_{1}}{\partial \alpha}=\sum_{c=1}^{r_{x}}\left\langle\sum_{i=1}^{m_{x}}\left\{\left(m_{x}+1-i\right) \ln \left(H_{i(i) c}\right)-\frac{(i-1) \ln \left(H_{i(i) c}\right)}{\left(\left(H_{i(i) c}\right)^{-\alpha}-1\right)}\right\}\right\rangle+\frac{r_{x} m_{x}}{\alpha}, \\
& \frac{\partial \ln L_{1}}{\partial \beta}=\sum_{d=1}^{r_{y}}\left\langle\sum_{j=1}^{m_{y}}\left\{\left(m_{y}+1-j\right) \ln \left(O_{j(j) d}\right)-\frac{(j-1) \ln \left(O_{j(j) d}\right)}{\left(O_{j(j) d}^{-\beta}-1\right)}\right\}\right\rangle+\frac{r_{y} m_{y}}{\beta}, \\
& \frac{\partial \ln L_{1}}{\partial \lambda}=\frac{r_{x} m_{x}+r_{y} m_{y}}{\lambda}-\sum_{c=1}^{r}\left\{\sum_{i=1}^{m_{x}}\left[\left(x_{i(i) c}\right)^{-1}-\frac{\left[\alpha\left(m_{x}-i+1\right)-1\right] \mathrm{e}^{-\left(\lambda / x_{i(i) c}\right)}}{\left(H_{i(i) c}\right)\left(x_{i(i) c}\right)}+\frac{(i-1) \alpha H_{i(i) c}^{\alpha-1} \mathrm{e}^{-\left(\lambda / x_{i(i) c}\right)}}{\left(x_{i(i) c}\right)\left(1-H_{i(i) c}^{\alpha}\right)}\right]\right\} \\
&-\sum_{d=1}^{r_{y}}\left\{\sum_{j=1}^{m_{y}}\left[\frac{1}{y_{j(j) d}}-\frac{\left[\beta\left(m_{y}+1-j\right)-1\right] \mathrm{e}^{-\left(\lambda / y_{j(j) d}\right)}}{\left(O_{j(j) d}\right)\left(y_{j(j) d}\right)}+\frac{(j-1) \beta\left(O_{j(j) d}\right)^{\beta-1} \mathrm{e}^{-\left(\lambda / y_{j(j) d}\right)}}{\left(y_{j(j) d}\right)\left(1-\left(O_{j(j) d}\right)^{\beta}\right)}\right]\right\} .
\end{aligned}
$$

An iterative technique is worked to solve Equations (11) -(13) numerically. Hence the ML estimators of $\alpha, \beta$ and $\lambda$ are obtained. According to invariance property the MSS reliability estimator is obtained after substituting these estimators in Equation (4).

\section{MSS RELIABILITY ESTIMATOR BASED ON MRSS}

Here, we obtain four estimators of $R_{s, k}$ when $X$ and $Y$ are independent GIE distributions using MRSS for odd set size (MRSSO) or MRSS for even set size (MRSSE). The first and second estimators are considered when both stress and strength data are selected from the same set sizes. The third and fourth estimators are regarded when both stress and strength data are selected from the different set sizes.

Here, the MSS reliability estimator is derived when $X \sim \operatorname{GIE}(\lambda, \alpha)$ and $Y \sim \operatorname{GIE}(\lambda, \beta)$ and their samples are selected from MRSSO. Let $X_{i(g) c} ; i=1, \ldots, m_{x}, c=1, \ldots, r_{x} ; g=\left[\left(m_{x}+1\right) / 2\right]$ be the available MRSSO selected from strength distribution. Also, suppose that $Y_{j(k) d} ; j=1, \ldots, m_{y}, d=1, \ldots, r_{y} ; k=\left[\left(m_{y}+1\right) / 2\right]$ be the available MRSSO chosen from stress distribution. The likelihood function $L_{2}$ of the observed samples is:

$$
\begin{aligned}
& L_{2}=\prod_{c=1}^{r_{x}} \prod_{i=1}^{m_{x}} \frac{m_{x} ! \lambda \alpha e^{-\lambda / x_{i(g) c}}}{[(g-1) !]^{2} x_{i(g) c}^{2}}\left[1-N_{i(g) c}^{\alpha}\right]^{g-1}\left(N_{i(g) c}\right)^{\alpha g-1} \prod_{d=1}^{r_{y}} \prod_{j=1}^{m_{y}} \frac{m_{y} ! \lambda \beta e^{-\lambda / y_{j(k) d}}}{[(k-1) !]^{2} y_{j(k) d}^{2}}\left(Z_{j(k) d}\right)^{\beta k-1}\left[1-\left(Z_{j(k) d}\right)^{\beta}\right]^{k-1}, \\
& N_{i(g) c}=\left[1-e^{-\left(\lambda / x_{i(g) c}\right)}\right] \text { and } Z_{j(k) d}=\left[1-e^{-\left(\lambda / y_{j(k) d}\right)}\right] . \text { So, the log-likelihood function of } L_{2} \text { is }
\end{aligned}
$$




$$
\begin{aligned}
\ln L_{2} & \propto r_{x} m_{x} \ln (\lambda \alpha)-\sum_{c=1}^{r_{x}}\left\langle\sum_{i=1}^{m_{x}}\left[2 \ln \left(x_{i(g) c}\right)-(g-1) \ln \left[1-N_{i(g) c}^{\alpha}\right]+\frac{\lambda}{x_{i(g) c}}\right]\right\rangle \\
& +\sum_{c=1}^{r_{x}} \sum_{i=1}^{m_{x}}(\alpha g-1) \ln \left(N_{i(g) c}\right)-\sum_{d=1}^{r_{y}}\left\{\sum_{j=1}^{m_{y}}\left(2 \ln \left(y_{j(k) d}\right)+\frac{\lambda}{y_{j(k) d}}\right)\right\}+r_{y} m_{y} \ln (\lambda \beta) \\
& +\sum_{d=1}^{r_{y}}\left\langle\sum_{j=1}^{m_{y}}\left[(\beta k-1) \ln \left(Z_{j(k) d}\right)+(k-1) \ln \left(1-Z_{j(k) d}^{\beta}\right)\right]\right\rangle .
\end{aligned}
$$

The first partial derivatives of $\ln L_{2}$ related to parameters are derived below:

$$
\begin{aligned}
& \frac{\partial \ln L_{2}}{\partial \alpha}=\frac{r_{x} m_{x}}{\alpha}-\sum_{c=1}^{r_{x}} \sum_{i=1}^{m_{x}} \frac{(g-1) \ln N_{i(g) c}}{\left[N_{i(g) c}^{-\alpha}-1\right]}+\sum_{c=1}^{r_{x}} \sum_{i=1}^{m_{x}} g \ln \left(N_{i(g) c}\right), \\
& \frac{\partial \ln L_{2}}{\partial \beta}=\frac{r_{y} m_{y}}{\beta}-\sum_{d=1}^{r_{y}} \sum_{j=1}^{m_{y}} \frac{(k-1) \ln Z_{j(k) d}}{\left[Z_{j(k) d}^{-\beta}-1\right]}+\sum_{d=1}^{r_{y}} \sum_{j=1}^{m_{y}} k \ln \left(\mathrm{Z}_{j(k) d}\right),
\end{aligned}
$$

and,

$$
\begin{gathered}
\frac{\partial \ln L_{2}}{\partial \lambda}=\frac{r_{x} m_{x}+r_{y} m_{y}}{\lambda}-\sum_{c=1}^{r_{x}} \sum_{i=1}^{m_{x}}\left(x_{i(g) c}\right)^{-1}-\sum_{c=1}^{r_{x}} \sum_{i=1}^{m_{x}} \frac{(g-1) \alpha N_{i(g) c}^{\alpha-1} e^{-\lambda / x_{i(g) c}}}{x_{i(g) c}\left[1-N_{i(g) c}^{\alpha}\right]}+\sum_{c=1}^{r_{x}} \sum_{i=1}^{m_{x}} \frac{(\alpha g-1) e^{-\lambda / x_{i(g) c}}}{x_{i(g) c} N_{i(g) c}} \\
-\sum_{d=1}^{r_{y}} \sum_{j=1}^{m_{y}} \frac{(k-1) \beta Z_{j(k) d}^{\beta-1} e^{-\lambda / y_{j(k) d}}}{\left[1-Z_{j(k) d}^{\beta}\right] y_{j(k) d}}+\sum_{d=1}^{r_{y}} \sum_{j=1}^{m_{y}} \frac{(\beta k-1) e^{-\lambda / y_{j(k) d}}}{y_{j(k) d} Z_{j(k) d}}-\sum_{d=1}^{r_{y}} \sum_{j=1}^{m_{y}} y_{j(k) d}^{-1}
\end{gathered}
$$

Solving numerically Equations (14)-(16), we get the ML estimators of $\alpha, \beta$ and $\lambda$ based on MRSS. Inserting the ML estimators of $\alpha$ and $\beta$ in Equation (4) we get the MSS reliability estimator.

\subsection{MSS Reliability Estimator with Even Set Size}

We regard the MSS estimator, where $X \sim \operatorname{GIE}(\lambda, \alpha)$ and $Y \sim$ GIE using MRSSE. Let $\left[\left\{X_{i(q) c}, i=1, \ldots, q\right\} \cup\right.$ $\left.\left\{X_{i(q+1) c}, i=q+1, \ldots, m_{x}\right\} ; c=1, \ldots, r_{x}, q=m_{x} / 2\right]$ be the MRSSE chosen from strength distribution. Also, let $\left[\left\{Y_{j(v) d}, j=1, \ldots, v\right\} \cup\left\{Y_{j(v+1) d}, j=v+1, \ldots, m_{y}\right\} ; d=1, \ldots, r_{y}, v=m_{y} / 2\right]$ be the MRSSE chosen from the stress distribution. The likelihood function $L_{3}$ is given by

$$
\begin{aligned}
L_{3}= & \prod_{c=1}^{r_{x}} \prod_{i=1}^{q} \frac{m_{x} ! \lambda \alpha e^{-\lambda / x_{i(q) c}}}{(q-1) ! q !\left(x_{i(q) c}^{2}\right)}\left[1-\left\{1-e^{-\left(\lambda / x_{i(q) c}\right)}\right\}^{\alpha}\right]^{q-1}\left[1-e^{-\left(\lambda / x_{i(q) c}\right)}\right]^{\alpha q+\alpha-1} \\
& \prod_{c=1}^{r_{x}} \prod_{i=q+1}^{m_{x}} \frac{m_{x} ! \lambda \alpha e^{-\lambda / x_{i(q+1) c}}}{(q-1) ! q !\left(x_{i(q+1) c}^{2}\right)}\left[1-\left\{1-e^{-\left(\lambda / x_{i(q+1) c}\right)}\right\}^{\alpha}\right]^{q}\left[1-e^{-\left(\lambda / x_{i(q+1) c}\right)}\right]^{\alpha q-1} \\
& \prod_{d=1}^{r_{y}} \prod_{j=1}^{v} \frac{m_{y} ! \lambda \beta e^{-\lambda / y_{j(v) d}}}{(v-1) ! v ! y_{j(v) d}^{2}}\left[1-\left\{1-e^{-\left(\lambda / y_{j(v) d}\right)}\right\}^{\beta}\right]^{\nu-1}\left[1-e^{-\left(\lambda / y_{j(v) d}\right)}\right]^{\beta v+\beta-1} \\
& \prod_{d=1}^{r_{y}} \prod_{j=v+1}^{m_{y}} \frac{m_{y} ! \lambda \beta e^{-\lambda / y_{j(v+1) d}}}{(v-1) ! v !\left(y_{j(v+1) d}^{2}\right)}\left[1-\left\{1-e^{-\left(\lambda / y_{j(v+1) d}\right)}\right\}^{\beta}\right]^{v}\left[1-e^{-\left(\lambda / y_{j(v+1) d}\right)}\right]^{\beta v-1} .
\end{aligned}
$$

The log-likelihood function of $L_{3}$ for $\alpha, \beta$ and $\lambda$ depending on the observed MRSSE is 


$$
\begin{aligned}
& \ln L_{3} \propto m_{x} r_{x} \ln (\lambda \alpha)-\sum_{c=1}^{r_{x}}\left\{\sum_{i=1}^{q} \frac{\lambda}{x_{i(q) c}}+\sum_{i=q+1}^{m_{x}} \frac{\lambda}{x_{i(q+1) c}}\right\}+\sum_{c=1}^{r_{x}}\left(A_{1}+A_{2}\right)-2 \sum_{c=1}^{r_{x}}\left\{\sum_{i=1}^{q} x_{i(q) c}+\sum_{i=q+1}^{m_{x}} x_{i(q+1) c}\right\} \\
& +m_{y} r_{y} \ln (\lambda \beta)-\sum_{d=1}^{r_{v}}\left\langle\sum_{j=1}^{v} \frac{\lambda}{y_{j(v) d}}+\sum_{j=v+1}^{m_{y}} \frac{\lambda}{y_{j(v+1) d}}\right\rangle+\sum_{d=1}^{r_{y}}\left(T_{1}+T_{2}\right)-2 \sum_{d=1}^{r_{v}}\left\langle\sum_{j=1}^{v} \ln y_{j(v) d}+\sum_{j=v+1}^{m_{y}} \ln y_{j(v+1) d}\right\rangle \text {, } \\
& A_{1}=\sum_{i=1}^{q}(q-1) \ln \left[1-\left(1-e^{-\left(\lambda / x_{i(q) c}\right)}\right)^{\alpha}\right]+\sum_{i=q+1}^{m_{x}} q \ln \left[1-\left(1-e^{-\left(\lambda / x_{i(q+1) c}\right)}\right)^{\alpha}\right] \text {, } \\
& A_{2}=\sum_{i=1}^{q}(\alpha q-1+\alpha) \ln \left(1-e^{-\left(\lambda / x_{i(q) c}\right)}\right)+\sum_{i=q+1}^{m_{x}}(\alpha q-1) \ln \left(1-e^{-\left(\lambda / x_{i(q+1) c}\right)}\right), \\
& T_{1}=\sum_{j=1}^{v}(v-1) \ln \left\{1-\left(1-e^{-\left(\lambda / y_{j(v) d}\right)}\right)^{\beta}\right\}+\sum_{j=v+1}^{m_{y}} v \ln \left[1-\left(1-e^{-\left(\lambda / y_{j(v+1) d}\right)}\right)^{\beta}\right] \text {, } \\
& T_{2}=\left[\sum_{j=1}^{v}(\beta v+\beta-1) \ln \left(1-e^{-\left(\lambda / y_{j(v) d}\right)}\right)+\sum_{j=v+1}^{m_{y}}(\beta v-1) \ln \left(1-e^{-\left(\lambda / y_{j(v+1) d}\right)}\right)\right] .
\end{aligned}
$$

The partial derivatives of $L_{3}$ regard to $\alpha, \beta$ and $\lambda$ are obtained as follows:

$$
\begin{aligned}
& \frac{\partial \ln L_{3}}{\partial \alpha}=\frac{m_{x} r_{x}}{\alpha}-\sum_{c=1}^{r_{x}}\left[\sum_{i=1}^{q} \frac{(q-1) \ln \left(1-e^{-\left(\lambda / x_{i(q) c}\right)}\right)}{\left(1-e^{-\left(\lambda / x_{i(q) c}\right)}\right)^{-\alpha}-1}+\sum_{i=q+1}^{m_{x}} \frac{q \ln \left(1-e^{-\left(\lambda / x_{i(q+1) c}\right)}\right)}{\left(1-e^{-\left(\lambda / x_{i(q+c) c}\right)}\right)^{-\alpha}-1}\right]+ \\
& \sum_{c=1}^{r_{x}}\left[\sum_{i=1}^{q}(q+1) \ln \left(1-e^{-\left(\lambda / x_{i(q) c}\right)}\right)+\sum_{i=q+1}^{m_{x}} q \ln \left(1-e^{-\left(\lambda / x_{i(q+1) c}\right)}\right)\right] \text {, } \\
& \frac{\partial \ln L_{3}}{\partial \beta}=\frac{m_{y} r_{y}}{\beta}-\sum_{d=1}^{r_{y}}\left[\sum_{j=1}^{v} \frac{(v-1) \ln \left(1-e^{-\left(\lambda / y_{j(v) d}\right)}\right)}{\left(1-e^{-\left(\lambda / y_{j(v) d}\right)}\right)^{-\beta}-1}+\sum_{j=v+1}^{m_{y}} \frac{v \ln \left(1-e^{-\left(\lambda / y_{j(v+1) d}\right)}\right)}{\left(\left(1-e^{-\left(\lambda / y_{j(v+1) d}\right)}\right)^{-\beta}-1\right.}\right]+ \\
& \sum_{d=1}^{r_{y}}\left[\sum_{j=1}^{v}(v+1) \ln \left(1-e^{-\left(\lambda / y_{j(v) d}\right)}\right)+\sum_{j=v+1}^{m_{y}} v \ln \left(1-e^{-\left(\lambda / y_{j(v+1) d}\right)}\right)\right] \text {, } \\
& \frac{\partial \ln L_{3}}{\partial \lambda}=\frac{m_{x} r_{x}+m_{y} r_{y}}{\lambda}-\sum_{c=1}^{r_{x}}\left\langle\sum_{i=1}^{q} \frac{(q-1) \alpha e^{-\lambda / x_{i(q) c}}\left(1-e^{-\lambda / x_{i(q) c}}\right)^{\alpha-1}}{x_{i(q) c}\left(1-\left(1-e^{-\lambda / x_{i(q) c}}\right)^{\alpha}\right)}+\sum_{i=q+1}^{m_{x}} \frac{q \alpha e^{-\lambda / x_{i(q+1) c}}\left(1-e^{-\lambda / x_{i(q+1) c}}\right)^{\alpha-1}}{x_{i(q+1) c}\left(1-\left(1-e^{-\lambda / x_{i(q+1) c}}\right)^{\alpha}\right)}\right\rangle- \\
& \sum_{c=1}^{r_{x}}\left(\sum_{i=1}^{q} x_{i(q) c}^{-1}+\sum_{i=q+1}^{m_{x}} x_{i(q+1) c}^{-1}\right)-\sum_{d=1}^{r_{y}}\left[\sum_{j=1}^{v} y_{j(v) d}^{-1}+\sum_{j=v+1}^{m_{y}} y_{j(v+1) d}^{-1}\right]- \\
& \sum_{d=1}^{r_{y}}\left\langle\sum_{j=1}^{v} \frac{(v-1)\left(1-e^{-\lambda / y_{j(v) d}}\right)^{\beta-1} \beta e^{-\lambda / y_{j(v) d}}}{y_{j(v) d}\left(1-\left(1-e^{-\left(\lambda / y_{j(v) d}\right)}\right)^{\beta}\right)}+\sum_{j=v+1}^{m_{y}} \frac{v \beta e^{-\left(\lambda / y_{j(v+1) d}\right)}\left(1-e^{-\left(\lambda / y_{j(v+1) d}\right)}\right)^{\beta-1}}{\left(1-\left(1-e^{-\left(\lambda / y_{j(v+1) d}\right)}\right)^{\beta}\right) y_{j(v+1) d}}\right\rangle \text {. }
\end{aligned}
$$

We solve numerically Equations (17)-(19) to get the ML estimators of $\alpha, \beta$ and $\lambda$. Hence, the ML of MSS reliability is obtained from Equation (4).

\subsection{MSS Reliability Estimator with Odd \&Even Set Sizes}

Here, the MSS reliability estimator is discussed, where $X \sim \operatorname{GIE}(\lambda, \alpha)$ under MRSSO. In contrast, we observe the random samples of $Y \sim \operatorname{GIE}(\lambda, \beta)$ under MRSSE. 
Let $X_{i(g) c} ; i=1, \ldots, m_{x}, g=\left[\left(m_{x}+1\right) / 2\right], c=1, \ldots, r_{x} ; \quad$ be the required MRSSO selected from strength distribution. Let $\left\{Y_{j(v) d} ; j=1, \ldots, v\right\} \cup\left\{Y_{j(v+1) d} ; j=v+1, \ldots, m_{\mathrm{y}}\right\} . ; d=1, \ldots, r_{y}$ be the required MRSSE extracted from stress distribution. Therefore, the likelihood function is

$$
\begin{aligned}
L_{4}= & \prod_{c=1}^{r_{x}} \prod_{i=1}^{m_{x}} \frac{m_{x} ! \lambda \alpha e^{-\lambda / x_{i(g) c}}}{[(g-1) !]^{2} x_{i(g) c}^{2}}\left\langle N_{i(g) c}\right\rangle^{\alpha g-1}\left\{1-N_{i(g) c}^{\alpha}\right\}^{g-1} \\
& \prod_{d=1}^{r_{y}} \prod_{j=1}^{v} \frac{m_{y} ! \lambda \beta e^{-\left(\lambda / y_{j(v) d}\right)}}{(v-1) ! v ! y_{j(v) d}^{2}}\left[1-\left(1-e^{-\left(\lambda / y_{j(v) d}\right)}\right)^{\beta}\right]^{\nu-1}\left(1-e^{-\left(\lambda / y_{j(v) d}\right)}\right)^{\beta \nu+\beta-1} \\
& \prod_{d=1}^{r_{y}} \prod_{j=v+1}^{m_{y}} \frac{m_{y} ! \lambda \beta e^{-\left(\lambda / y_{j(v+1) d}\right)}}{(v-1) ! v !\left(y_{j(v+1) d}^{2}\right)}\left\{1-e^{-\left(\lambda / y_{j(v+1) d}\right)}\right\}^{\beta v-1}\left\langle 1-\left[1-e^{-\left(\lambda / y_{j(v+1) d}\right)}\right]^{\beta}\right\rangle^{v} .
\end{aligned}
$$

The log-likelihood is as follows:

$$
\begin{aligned}
\ln L_{4} & \propto r_{x} m_{x} \ln (\lambda \alpha)-\sum_{c=1}^{r_{x}}\left\langle\sum_{i=1}^{m_{x}}\left[\frac{\lambda}{x_{i(g) c}}+2 \ln \left(x_{i(g) c}\right)\right]\right\rangle+\sum_{c=1}^{r_{x}}\left\langle\sum_{i=1}^{m_{x}}\left[(g-1) \ln \left(1-N_{i(g) c}^{\alpha}\right)+(\alpha g-1) \ln \left(N_{i(g) c}\right)\right]\right\rangle \\
+ & m_{y} r_{y} \ln (\lambda \beta)-\lambda \sum_{d=1}^{r_{y}}\left[\sum_{j=1}^{v} y_{j(v) d}^{-1}+\sum_{j=v+1}^{m_{y}} y_{j(v+1) d}^{-1}\right]+\sum_{d=1}^{r_{y}}\left(T_{1}+T_{2}\right)-2 \sum_{d=1}^{r_{y}}\left[\sum_{j=1}^{v} \ln y_{j(v) d}+\sum_{j=v+1}^{m_{y}} \ln y_{j(v+1) d}\right] .
\end{aligned}
$$

The partial derivatives of $\ln L_{4}$ related to $\alpha$ and $\beta$ are derived in Equations (14) and (18) respectively. The first partial derivative of $\ln L_{4}$ related to $\lambda$ is as follows:

$$
\begin{aligned}
& \frac{\partial \ln L_{4}}{\partial \lambda}=\frac{r_{x} m_{x}+r_{y} m_{y}}{\lambda}-\sum_{c=1}^{r_{x}} \sum_{i=1}^{m_{x}} \frac{1}{x_{i(g) c}}-\sum_{c=1}^{r_{x}} \sum_{i=1}^{m_{x}} \frac{(g-1) \alpha N_{i(g) c}^{\alpha-1} e^{-\lambda / x_{i(g) c}}}{x_{i(g) c}\left[1-N_{i(g) c}^{\alpha}\right]}+\sum_{c=1}^{r_{x}} \sum_{i=1}^{m_{x}} \frac{(\alpha g-1) e^{-\lambda / x_{i(g) c}}}{x_{i(g) c} N_{i(g) c}}- \\
& -\sum_{d=1}^{r_{y}}\left[\sum_{j=1}^{v} y_{j(v) d}^{-1}+\sum_{j=v+1}^{m_{y}} y_{j(v+1) d}^{-1}\right]-\sum_{d=1}^{r_{y}}\left(\sum_{j=1}^{v} \frac{(v-1) \beta\left(1-e^{\left.-\lambda / y_{j(v) d}\right)^{\beta-1}} e^{-\lambda / y_{j(v) d}}\right.}{y_{j(v) d}\left(1-\left(1-e^{-\lambda / y_{j(v) d}}\right)^{\beta}\right)}+\sum_{j=v+1}^{m_{y}} \frac{e^{-\left(\lambda / y_{j(v+1) d}\right)} v \beta\left(1-e^{-\left(\lambda / y_{j(v+1) d}\right)}\right)^{\beta-1}}{\left(1-\left(1-e^{-\left(\lambda / y_{j(v+1) d}\right)}\right)^{\beta}\right) y_{j(v+1) d}}\right) .
\end{aligned}
$$

Equations (14), (18) and (20) have no closed form solutions, so we employ the numerical technique to get the solution. Further, the MSS reliability estimator is yielded from Equation (4).

\subsection{MSS Reliability Estimator with Even \&Odd Set Sizes}

Here, we derive the MSS reliability estimator when the data of the strength are taken from the GIE distribution based on MRSSE. At the same time, the data of stress are taken from the GIE distribution from MRSSO. Let $\left\{X_{i(q) c}, i=1, \ldots, q\right\} \cup\left\{X_{i(q+1) c}, i=q+1, \ldots, m_{\mathrm{x}}\right\} ; c=1, \ldots, r_{x}$ be a MRSSE selected from GIE $(\lambda, \alpha)$ distribution. Let $Y_{j(k) d} ; j=1, \ldots, m_{y}, d=1, \ldots, r_{y} ; k=\left[\left(m_{y}+1\right) / 2\right]$ be the observed MRSSO drawn from the $\operatorname{GIE}(\lambda, \beta)$ distribution. The likelihood function, is given by

$$
\begin{aligned}
L_{5}= & \prod_{c=1}^{r_{x}} \prod_{i=1}^{q} \frac{m_{x} ! \lambda \alpha e^{-\left(\lambda / x_{i(q) c}\right)}}{(q-1) ! q !\left(x_{i(q) c}^{2}\right)}\left(1-e^{-\left(\lambda / x_{i(q) c}\right)}\right)^{\alpha q+\alpha-1}\left\langle 1-\left(1-e^{-\left(\lambda / x_{i(q) c}\right)}\right)^{\alpha}\right\rangle^{q-1} \\
& \prod_{c=1}^{r_{x}} \prod_{i=q+1}^{m_{x}} \frac{m_{x} ! \lambda \alpha e^{-\left(\lambda / x_{i(q+1) c}\right)}}{(q-1) ! q !\left(x_{i(q+1) c}^{2}\right)}\left(1-e^{-\left(\lambda / x_{i(q+1) c}\right)}\right)^{\alpha q-1}\left\langle 1-\left(1-e^{-\left(\lambda / x_{i(q+1) c}\right)}\right)^{\alpha}\right\rangle^{q} \\
& \prod_{d=1}^{r_{y}} \prod_{j=1}^{m_{y}} \frac{m_{y} ! \lambda \beta e^{-\left(\lambda / y_{j(k) d}\right)} Z_{j(k) d}^{\beta k-1}}{[(k-1) !]^{2}\left(y_{j(k) d}^{2}\right)}\left(1-Z_{j(k) d}^{\beta}\right)^{k-1} \cdot
\end{aligned}
$$


The log-likelihood function is

$$
\begin{aligned}
\ln L_{5} \propto m_{x} r_{x} \ln (\lambda \alpha)-\lambda \sum_{c=1}^{r_{x}}\left\langle\sum_{i=1}^{q} x_{i(q) c}^{-1}+\sum_{i=q+1}^{m_{x}} x_{i(q+1) c}^{-1}\right\rangle-2 \sum_{c=1}^{r_{x}}\left\langle\sum_{i=1}^{q} x_{i(q) c}+\sum_{i=q+1}^{m_{x}} x_{i(q+1) c}\right\rangle+\sum_{c=1}^{r_{x}}\left(A_{1}+A_{2}\right) \\
+r_{y} m_{y} \ln (\lambda \beta)-\sum_{d=1}^{r_{y}}\left\langle\sum_{j=1}^{m_{y}}\left[2 \ln \left(y_{j(k) d}\right)+\lambda y_{j(k) d}^{-1}\right]\right\rangle+\sum_{d=1}^{r_{y}}\left\langle\sum_{j=1}^{m_{y}}\left[(\beta k-1) \ln \left(Z_{j(k) d}\right)+(k-1) \ln \left[1-Z_{j(k) d}^{\beta}\right]\right]\right\rangle .
\end{aligned}
$$

The partial derivatives of $\beta$ and $\alpha$ are derived in (15) and (17) respectively. Also, we obtain the first derivative of $\lambda$ as follows:

$$
\begin{aligned}
\frac{\partial \ln L_{5}}{\partial \lambda} & =\frac{m_{x} r_{x}+m_{y} r_{y}}{\lambda}-\alpha \sum_{c=1}^{r_{x}}\left[\sum_{i=1}^{q} \frac{(q-1) e^{-\left(\lambda / x_{i(q) c}\right)}\left(1-e^{-\left(\lambda / x_{i(q) c}\right)}\right)^{\alpha-1}}{x_{i(q) c}\left(1-e^{-\left(\lambda / x_{i(q) c}\right)}\right)^{\alpha}}+\sum_{i=q+1}^{m_{x}} \frac{q e^{-\left(\lambda / x_{i(q+1) c}\right)}\left(1-e^{-\left(\lambda / x_{i(q+1) c}\right)}\right)^{\alpha-1}}{x_{i(q+1) c}\left(1-e^{-\left(\lambda / x_{i(q+1) c}\right)}\right)^{\alpha}}\right] \\
& -\sum_{c=1}^{r_{x}}\left(\sum_{i=1}^{q} x_{i(q) c}^{-1}+\sum_{i=q+1}^{m_{x}} x_{i(q+1) c}^{-1}\right)+\sum_{d=1}^{r_{y}} \sum_{j=1}^{m_{y}} \frac{(\beta k-1) e^{-\left(\lambda / y_{j(k) d}\right)}}{y_{j(k) d} Z_{j(k) d}}-\sum_{d=1}^{r_{y}}\left(\sum_{j=1}^{m_{y}}\left[y_{j(k) d}^{-1}+\frac{Z_{j(k) d}^{\beta-1} e^{-\left(\lambda / y_{j(k) d}\right)}(k-1) \beta}{\left[1-Z_{j(k) d}^{\beta}\right] y_{j(k) d}}\right]\right) .
\end{aligned}
$$

Solving Equations (15), (17) and (21) numerically, we find the ML estimators of $\alpha, \beta$ and $\lambda$. Hence, the MSS reliability estimator is yielded from Equation (4).

\section{SIMULATION STUDY}

A comprehensive illustration is utilized to compare the behaviour of $R_{s, k}$ estimates using SRS, RSS and MRSS. We consider four issues of stress and strength random variables under MRSS. The simulation study is designed as follows:

\$ 1000 random samples of sizes $(n, m)=(10,10),(10,15),(15,10),(15,15),(15,20),(20,15),(20$, $20),(25,25),(30,30),(35,35)$ are generated from the strength $X \sim \operatorname{GIE}(\lambda, \alpha)$ and stress $Y \sim \operatorname{GIE}$ $(\lambda, \beta)$ distributions based on SRS.

1000 random samples of set sizes $\left(m_{x}, m_{y}\right)=(2,2),(2,3),(3,2),(3,3),(3,4),(4,3),(4,4),(5,5),(6$, $6),(7,7)$, where the number of cycles is $r_{x}=r_{y}=r=5$, are generated from stress and strength populations based on RSS. A random sample $\left\{\left(X_{1(1) c}, X_{2(2) c}, \ldots X_{n(n) c}\right), c=1, \ldots, 5\right\}$ of size $(n=$ $\left.m_{x} r_{\mathrm{X}}=m_{x} r\right)$ is drawn from strength population. Also, a random sample $\left\{\left(Y_{1(1) d}, Y_{2(2) d}, \ldots Y_{m(m) d}\right), d\right.$ $=1, \ldots, 5\}$ of size $\left(m=m_{y} r_{y}=m_{y} r\right)$ is drawn from stress population.

* 1000 random samples of set sizes $\left(m_{x}, m_{y}\right)=(3,3),(5,5),(7,7)$, where the number of cycles is $r_{x}=$ $r_{y}=r=5$, are generated from $X$ and $Y$ populations based on MRSSO. Let $n=m_{x} r_{x}$ and $m=m_{y} r_{y}$ are the available random samples from $X$ and $Y$, where $\left\{X_{1(g) c}, X_{2(g) c}, X_{n(g) c}, c=1 \ldots 5, g=\left(m_{x}+1\right) / 2\right\}$ and $\left\{Y_{1(k) d}, Y_{2(k) d}, Y_{m(k) d}, d=1,2, \ldots 5, k=\left(m_{y}+1\right) / 2\right\}$.

1000 random samples of set sizes $\left(m_{x}, m_{y}\right)=(2,2),(4,4),(6,6)$, where the number of cycles is $r_{x}=$ $r_{y}=r=5$, are generated from $X$ and $Y$ populations based on MRSSE. A random sample of size $(n=$ $\left.m_{x} r_{x}=m_{x} r\right)$ and $\left(m=m_{y} r_{y}=m_{y} r\right)$ is drawn from $X$ and $Y$ as follows $\left\{X_{1(q) c}, X_{2(q) c}, \ldots, X_{q(q) c}, X_{q+1(q+1) c}\right.$, $\left.X_{q+2(q+1) c}, \ldots, X_{n(q+1) c}\right\}$ and $\left\{Y_{1(v) d}, Y_{2(v) d}, \ldots, Y_{v(v) d}, Y_{v+1(v+1) d}, Y_{v+2(v+1) d}, \ldots, Y_{m(v+1) d}\right\}$ where $q=m_{x} / 2$ and $v=m_{y} / 2$.

$*$ Values of $(\alpha, \beta)$ are chosen as $(0.5,1),(0.5,2.5),(1,3.5),(1.5,2.5)$ and $\lambda=0.5$.

* The true values of $R_{s, k}$ at $(s, k)=(1,3)$ are equal to $0.869,0.9,0.963$ and 0.982 and the true values of system reliability $R_{s, k}$ at $(s, k)=(2,4)$ are equal to $0.752,0.8,0.91$ and 0.952 .

* The ML estimate (MLE) of $\lambda$ is obtained using (10), then substituting the MLE of $\lambda$ in Equation (9), we get the MLEs of $\alpha$ and $\beta$. Then, inserting the MLE of population parameter in Equation (4), we obtain the MLE of $R_{s, k}$ in the case of SRS technique. 
* The MLEs of $\lambda, \alpha$ and $\beta$ based on RSS are obtained by solving numerically Equations (11) - (13). Then, the MLE of $R_{s, k}$ is yielded from Equation (4).

$*$ The MLEs of $\lambda, \alpha$ and $\beta$ are obtained in four cases based on MRSS. Then, the MLE of $R_{s, k}$ is yielded from Equation (4).

* In comparison, we compute the mean squared errors (MSEs) and relative efficiencies (REs) measures, where the RE of the RSS and MRSS relative to SRS are defined as:

$$
R E_{1}=\frac{\operatorname{MSE}\left(\hat{R}_{s, k}\left(Y_{S R S}<X_{S R S}\right)\right)}{\operatorname{MSE}\left(\hat{R}_{s, k}\left(Y_{R S S}<X_{R S S}\right)\right)}, \quad R E_{2}=\frac{\operatorname{MSE}\left(\hat{R}_{s, k}\left(Y_{S R S}<X_{S R S}\right)\right)}{\operatorname{MSE}\left(\hat{R}_{s, k}\left(Y_{M R S S}<X_{M R S S}\right)\right)} .
$$

All simulated studies are obtained via MathCAD 14. Tables 1-8 summarize the MSEs and REs of $R_{s, k}$ using the proposed sampling designs.

According to Tables $1-8$ and Figures 1-6, we remark the following:

- The MSEs of MSS reliability estimates under MRSS scheme, are preferable than the corresponding under SRS in majority of cases (Tables $1-8$ ).

- The MSEs of MSS reliability estimates via RSS scheme are smaller than the corresponding MSEs of MSS reliability estimates under SRS in majority of cases (Tables 1-8).

- The MSEs of the MSS estimate via RSS are less than the corresponding under MRSS and SRS schemes for most cases (see Figures 1 and 2).

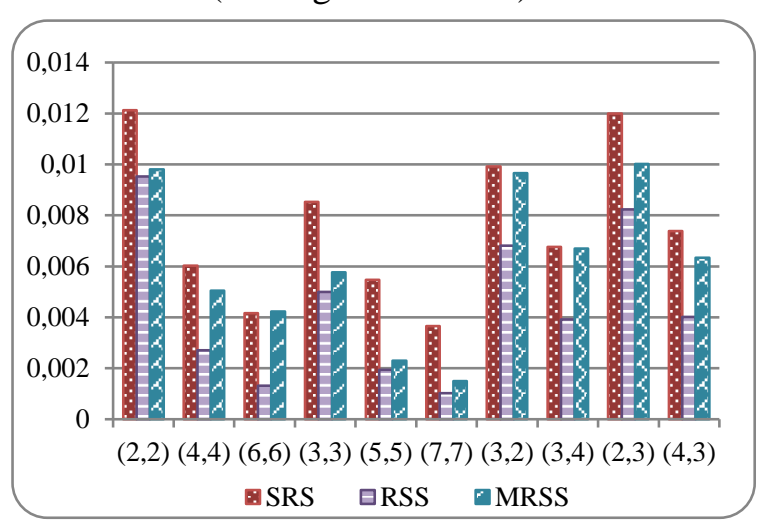

Figure 1. MSE of MSS estimate at $R_{2,4}=0.8$

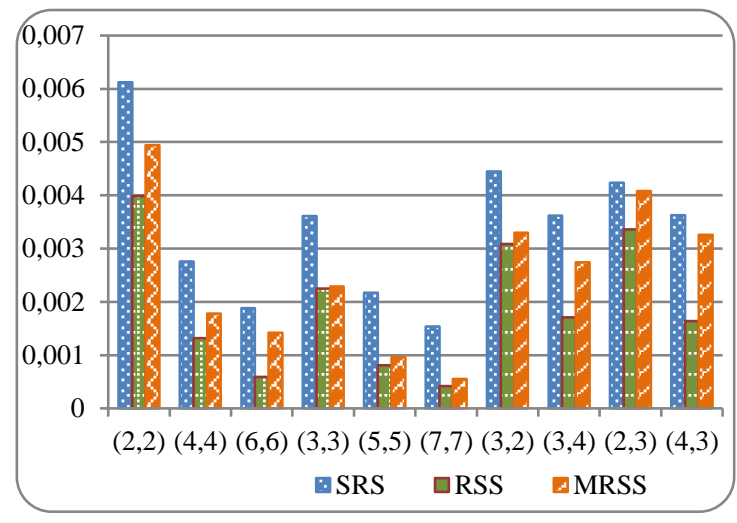

Figure 2. MSE of MSS estimate at $R_{1,3}=0.9$

- The MSEs of $R_{s, k}$ estimate for the considered sampling schemes decline as the exact value of $R_{s, k}$ increases for majority of situations (see Figures 3 and 4).

- Figures 5 and 6 show that the RSS method is preferable than MRSS method compared to SRS for most cases.

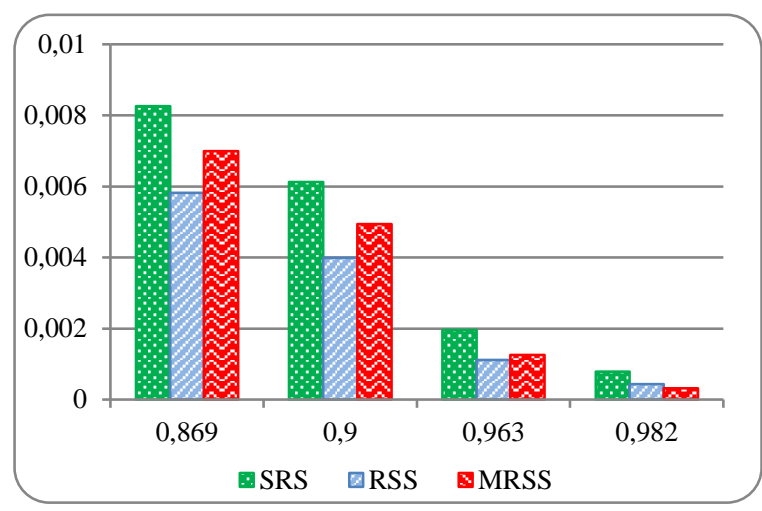

Figure 3. MSE of the MSS estimate at ( $m_{x}$, $\left.m_{y}\right)=(2,2)$ and $(s, k)=(1,3)$

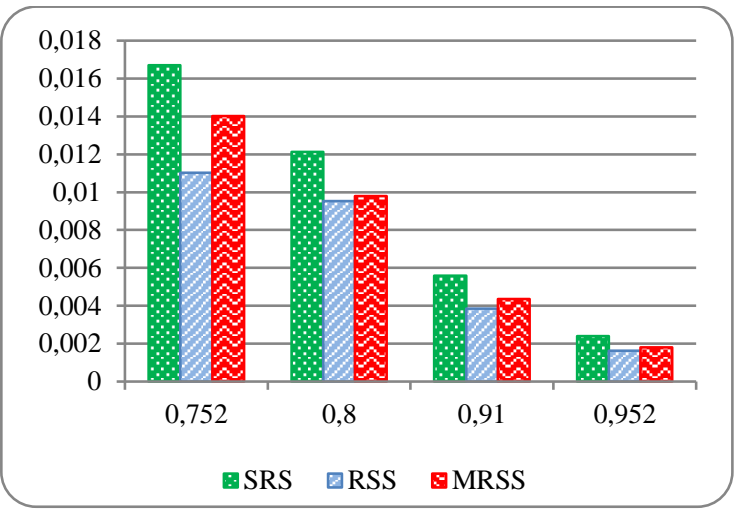

Figure 4. MSE of the MSS estimate at ( $m_{x}$, $\left.m_{y}\right)=(2,2)$ and $(s, k)=(2,4)$ 


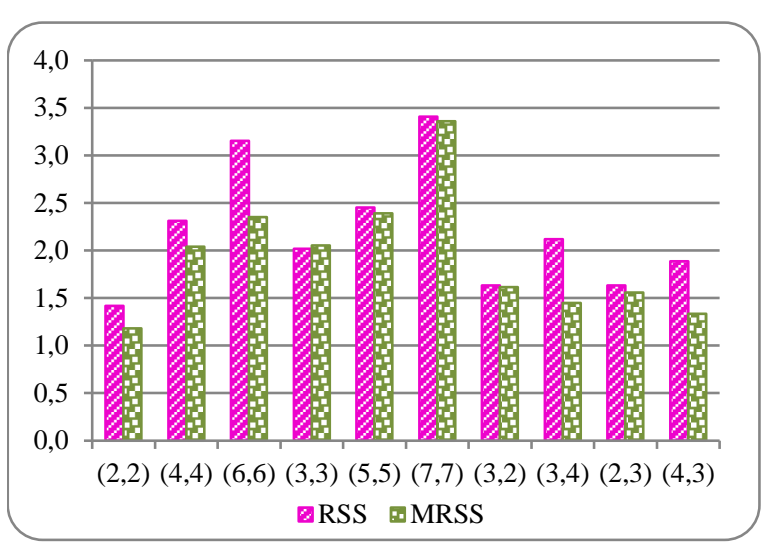

Figure 5. Efficiency of the MSS reliability estimates at $R_{1,3}=0.869$ under $R S S$ and MRSS with the corresponding SRS estimate

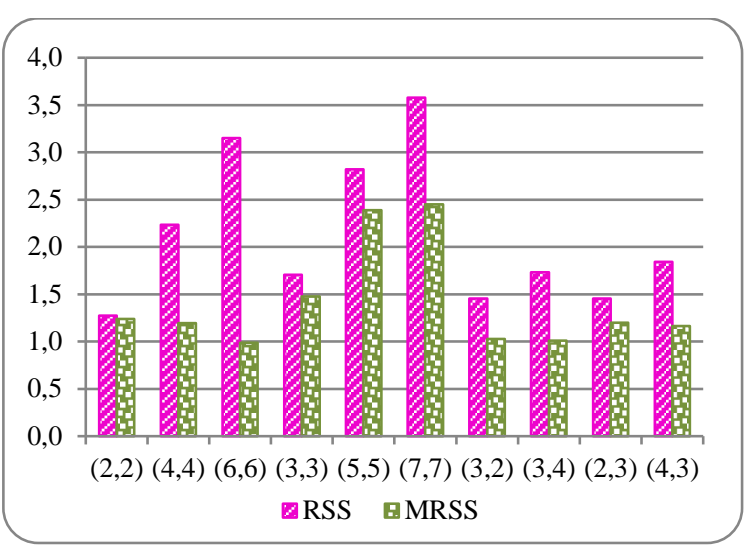

Figure 6. Efficiency of MSS reliability estimates at $R_{2,4}=0.8$ under RSS and MRSS with the corresponding SRS estimate

- The MSEs of $R_{s, k}$ under all schemes are decreasing with sample size. While the REs of $R_{s, k}$ under all schemes are increasing with sample size.

- The MSEs of MSS reliability estimates are decreasing as the value of $\beta$ increases for the same sample size and for a fixed value of $\alpha$, in approximately most of the situations. While, the MSEs of MSS reliability estimates are increasing as the value of $\alpha$ increases for the same sample size and for fixed value of $\beta$ in majority of the issues.

- $\quad$ This study posted that the MLEs of $R_{s, k}$ when $X$ and $Y$ are selected from MRSS are efficient than the MLEs of $R_{s, k}$ in the situation of ( $\left.X_{\mathrm{MRSS}} \& Y_{\mathrm{MRSS}}\right)$ and ( $\left.X_{\mathrm{RSS}} \& Y_{\mathrm{RSS}}\right)$. Also, the MLEs of $R_{s, k}$ using RSS are efficient than the MLEs in the situation of ( $\left.X_{\mathrm{MRSS}} \& Y_{\mathrm{MRSS}}\right)$ and $\left(X_{\mathrm{SRS}} \& Y_{\mathrm{SRS}}\right)$.

- Generally, the MSS estimates using RSS are efficient than the associated estimates under MRSS and SRS

Table 1. MSEs of $R_{s, k}$ estimates and their efficiencies based on SRS, RSS and MRSSE for 5 cycles, $(s, k)=(1$, 3)

\begin{tabular}{|c|c|c|c|c|c|c|}
\hline \multirow{2}{*}{$r$} & \multirow{2}{*}{$\left(m_{x}, m_{y}\right)$} & \multicolumn{3}{|l|}{ MSE } & \multirow{2}{*}{$\begin{array}{l}\text { RE }_{1} \\
\text { RSS }\end{array}$} & \multirow{2}{*}{$\begin{array}{l}\mathrm{RE}_{2} \\
\text { MRSSE }\end{array}$} \\
\hline & & SRS & RSS & MRSSE & & \\
\hline \multicolumn{7}{|c|}{$R_{s, k}=0.869,(\alpha, \beta)=(1.5,2.5)$} \\
\hline 5 & $(2,2)$ & 0.00826 & 0.00582 & 0.00699 & 1.419 & 1.182 \\
\hline 5 & $(4,4)$ & 0.00402 & 0.00174 & 0.00197 & 2.31 & 2.041 \\
\hline 5 & $(6,6)$ & 0.00268 & 0.00085 & 0.00114 & 3.153 & 2.351 \\
\hline \multicolumn{7}{|c|}{$R_{s, k}=0.9,(\alpha, \beta)=(0.5,1)$} \\
\hline 5 & $(2,2)$ & 0.00612 & 0.00399 & 0.00494 & 1.534 & 1.239 \\
\hline 5 & $(4,4)$ & 0.00276 & 0.00132 & 0.00178 & 2.091 & 1.551 \\
\hline 5 & $(6,6)$ & 0.00188 & 0.00059 & 0.00142 & 3.186 & 1.324 \\
\hline \multicolumn{7}{|c|}{$R_{s, k}=0.963,(\alpha, \beta)=(1,3.5)$} \\
\hline 5 & $(2,2)$ & 0.00196 & 0.00111 & 0.00126 & 1.766 & 1.556 \\
\hline 5 & $(4,4)$ & 0.00032 & 0.00030 & 0.00035 & 1.067 & 0.914 \\
\hline 5 & $(6,6)$ & 0.00048 & 0.00015 & 0.00029 & 3.200 & 1.655 \\
\hline \multicolumn{7}{|c|}{$R_{s, k}=0.982,(\alpha, \beta)=(0.5,2.5)$} \\
\hline 5 & $(2,2)$ & 0.00078 & 0.00043 & 0.00032 & 1.814 & 2.438 \\
\hline 5 & $(4,4)$ & 0.00022 & 0.00011 & 0.00012 & 2.000 & 1.833 \\
\hline 5 & $(6,6)$ & 0.00015 & 0.00005 & 0.00013 & 3.000 & 1.154 \\
\hline
\end{tabular}


Table 2. MSEs of Rs, $k$ estimates and their efficiencies based on SRS, RSS and MRSSO for 5 cycles, ( $s$, $k)=(1,3)$

\begin{tabular}{|l|l|l|l|l|l|l|}
\hline \multirow{2}{*}{$\boldsymbol{r}$} & \multirow{2}{*}{$\left(\boldsymbol{m}_{x}, \boldsymbol{m}_{y}\right)$} & MSE & $\mathbf{R E}_{\mathbf{1}}$ & $\mathbf{R E}_{\mathbf{2}}$ \\
\cline { 2 - 6 } & SRS & $\mathbf{R S S}$ & MRSSO & RSS & MRSSO \\
\hline$R_{s, k}=\mathbf{0 . 8 6 9},(\boldsymbol{\alpha}, \boldsymbol{\beta})=(\mathbf{1 . 5}, \mathbf{2 . 5})$ \\
\hline 5 & $(3,3)$ & 0.00606 & 0.00300 & 0.00295 & 2.020 & 2.054 \\
\hline 5 & $(5,5)$ & 0.00299 & 0.00122 & 0.00125 & 2.451 & 2.392 \\
\hline 5 & $(7,7)$ & 0.00225 & 0.00066 & 0.00067 & 3.409 & 3.358 \\
\hline$R_{s, k}=\mathbf{0 . 9},(\boldsymbol{\alpha}, \boldsymbol{\beta})=(\mathbf{0 . 5}, \mathbf{1})$ \\
\hline 5 & $(3,3)$ & 0.00361 & 0.00225 & 0.00229 & 1.604 & 1.576 \\
\hline 5 & $(5,5)$ & 0.00217 & 0.00081 & 0.00096 & 2.679 & 2.260 \\
\hline 5 & $(7,7)$ & 0.00154 & 0.00042 & 0.00055 & 3.667 & 2.800 \\
\hline$R_{s, k}=\mathbf{0 . 9 6 3 ,}(\boldsymbol{\alpha}, \boldsymbol{\beta})=(\mathbf{1}, \mathbf{3 . 5})$ \\
\hline 5 & $(3,3)$ & 0.00117 & 0.00054 & 0.00067 & 2.167 & 1.746 \\
\hline 5 & $(5,5)$ & 0.0006 & 0.00021 & 0.00025 & 2.857 & 2.400 \\
\hline 5 & $(7,7)$ & 0.00041 & 0.00011 & 0.00014 & 3.727 & 2.929 \\
\hline$R_{s, k}=\mathbf{0 . 9 8 2 ,}(\boldsymbol{\alpha}, \boldsymbol{\beta})=(\mathbf{0 . 5}, \mathbf{2 . 5})$ \\
\hline 5 & $(3,3)$ & 0.00036 & 0.00017 & 0.00019 & 2.118 & 1.895 \\
\hline 5 & $(5,5)$ & 0.00018 & 0.00007 & 0.00007 & 2.571 & 2.571 \\
\hline 5 & $(7,7)$ & 0.00015 & 0.00004 & 0.00004 & 3.750 & 3.750 \\
\hline
\end{tabular}

Table 3. MSEs of $R_{s, k}$ estimates and their efficiencies based on SRS, RSS and MRSS (X has odd set size and $Y$ has even set size) for 5 cycles, $(s, k)=(1,3)$

\begin{tabular}{|c|c|c|c|c|c|c|}
\hline \multirow{2}{*}{$\boldsymbol{r}$} & \multirow{2}{*}{$\left(m_{x}, m_{y}\right)$} & \multicolumn{3}{|l|}{ MSE } & \multirow{2}{*}{$\begin{array}{l}\mathrm{RE}_{1} \\
\mathrm{RSS}\end{array}$} & \multirow{2}{*}{$\begin{array}{l}\mathrm{RE}_{2} \\
\text { MRSS }\end{array}$} \\
\hline & & SRS & RSS & MRSS & & \\
\hline \multicolumn{7}{|c|}{$R_{s, k}=0.869,(\alpha, \beta)=(1.5,2.5)$} \\
\hline 5 & $(3,2)$ & 0.00653 & 0.00400 & 0.00404 & 1.633 & 1.616 \\
\hline 5 & $(3,4)$ & 0.00485 & 0.00229 & 0.00335 & 2.118 & 1.448 \\
\hline \multicolumn{7}{|c|}{$R_{s, k}=0.9,(\alpha, \beta)=(0.5,1)$} \\
\hline 5 & $(3,2)$ & 0.00445 & 0.00309 & 0.0033 & 1.440 & 1.348 \\
\hline 5 & $(3,4)$ & 0.00362 & 0.00171 & 0.00274 & 2.117 & 1.321 \\
\hline \multicolumn{7}{|c|}{$R_{s, k}=0.963,(\alpha, \beta)=(1,3.5)$} \\
\hline 5 & $(3,2)$ & 0.0012 & 0.00084 & 0.00066 & 1.429 & 1.818 \\
\hline 5 & $(3,4)$ & 0.00089 & 0.00046 & 0.00041 & 1.935 & 2.171 \\
\hline \multicolumn{7}{|c|}{$R_{s, k}=0.982,(\alpha, \beta)=(0.5,2.5)$} \\
\hline 5 & $(3,2)$ & 0.00052 & 0.00024 & 0.00014 & 2.167 & 3.714 \\
\hline 5 & $(3,4)$ & 0.00035 & 0.00014 & 0.00012 & 2.500 & 2.917 \\
\hline
\end{tabular}

Table 4. MSEs of $R_{s, k}$ estimates and their efficiencies based on SRS, RSS and MRSS (X has even set size and $Y$ has odd set size) for 5 cycles, $(s, k)=(1,3)$

\begin{tabular}{|c|c|c|c|c|c|c|}
\hline \multirow{2}{*}{$\boldsymbol{r}$} & \multirow{2}{*}{$\left(m_{x}, m_{y}\right)$} & \multicolumn{3}{|l|}{ MSE } & \multirow{2}{*}{$\begin{array}{l}\mathrm{RE}_{1} \\
\mathrm{RSS} \\
\end{array}$} & \multirow{2}{*}{$\begin{array}{l}\mathrm{RE}_{2} \\
\mathrm{MRSS} \\
\end{array}$} \\
\hline & & SRS & RSS & MRSS & & \\
\hline \multicolumn{7}{|c|}{$R_{s, k}=0.869,(\alpha, \boldsymbol{\beta})=(1.5,2.5)$} \\
\hline 5 & $(2,3)$ & 0.00777 & 0.00476 & 0.00499 & 1.632 & 1.557 \\
\hline 5 & $(4,3)$ & 0.00461 & 0.00224 & 0.00300 & 2.058 & 1.537 \\
\hline \multicolumn{7}{|c|}{$R_{s, k}=0.9,(\alpha, \beta)=(0.5,1)$} \\
\hline 5 & $(2,3)$ & 0.00424 & 0.00336 & 0.00408 & 1.262 & 1.039 \\
\hline 5 & $(4,3)$ & 0.00363 & 0.00164 & 0.00326 & 2.213 & 1.113 \\
\hline \multicolumn{7}{|c|}{$R_{s, k}=0.963,(\alpha, \beta)=(1,3.5)$} \\
\hline 5 & $(2,3)$ & 0.0015 & 0.00094 & 0.00086 & 1.596 & 1.744 \\
\hline 5 & $(4,3)$ & 0.00087 & 0.00042 & 0.00048 & 2.071 & 1.813 \\
\hline
\end{tabular}




\begin{tabular}{|l|l|l|l|l|l|l|}
\hline \multirow{2}{*}{$\boldsymbol{r}$} & \multirow{2}{*}{$\left(\boldsymbol{m}_{\boldsymbol{x}}, \boldsymbol{m}_{\boldsymbol{y}}\right)$} & MSE & \multicolumn{1}{|l|}{$\mathbf{R E}_{\boldsymbol{1}}$} & $\mathbf{R E}_{\boldsymbol{2}}$ \\
\cline { 3 - 7 } & SRS & RSS & MRSS & RSS & MRSS \\
\hline 5 & $(2,3)$ & 0.00059 & 0.00030 & 0.00026 & 1.967 & 2.269 \\
\hline 5 & $(4,3)$ & 0.00029 & 0.00013 & 0.00019 & 2.231 & 1.526 \\
\hline
\end{tabular}

Table 5. MSEs of $R_{s, k}$ estimates and their efficiencies based on SRS, RSS and MRSSE for 5 cycles, $(s, k)=(2$, 4)

\begin{tabular}{|c|c|c|c|c|c|c|}
\hline \multirow{2}{*}{$\boldsymbol{r}$} & \multirow{2}{*}{$\left(m_{x}, m_{y}\right)$} & \multicolumn{3}{|l|}{ MSE } & \multirow{2}{*}{$\begin{array}{l}\text { RE }_{1} \\
\text { RSS }\end{array}$} & \multirow{2}{*}{$\begin{array}{l}\mathbf{R E}_{2} \\
\text { MRSSE }\end{array}$} \\
\hline & & SRS & RSS & MRSSE & & \\
\hline \multicolumn{7}{|c|}{$R_{s, k}=0.752,(\alpha, \beta)=(1.5,2.5)$} \\
\hline 5 & $(2,2)$ & 0.01671 & 0.01102 & 0.01402 & 1.516 & 1.192 \\
\hline 5 & $(4,4)$ & 0.00735 & 0.00353 & 0.00480 & 2.082 & 1.531 \\
\hline 5 & $(6,6)$ & 0.00524 & 0.00162 & 0.00265 & 3.235 & 1.977 \\
\hline \multicolumn{7}{|c|}{$R_{s, k}=0.8,(\alpha, \beta)=(0.5,1)$} \\
\hline 5 & $(2,2)$ & 0.01213 & 0.00953 & 0.00980 & 1.273 & 1.238 \\
\hline 5 & $(4,4)$ & 0.00603 & 0.0027 & 0.00505 & 2.233 & 1.194 \\
\hline 5 & $(6,6)$ & 0.00416 & 0.00132 & 0.00422 & 3.152 & 0.986 \\
\hline \multicolumn{7}{|c|}{$R_{S, k}=0.91,(\alpha, \beta)=(1,3.5)$} \\
\hline 5 & $(2,2)$ & 0.0056 & 0.00384 & 0.00436 & 1.458 & 1.284 \\
\hline 5 & $(4,4)$ & 0.00273 & 0.00123 & 0.00161 & 2.220 & 1.696 \\
\hline 5 & $(6,6)$ & 0.00180 & 0.00061 & 0.00136 & 2.951 & 1.324 \\
\hline \multicolumn{7}{|c|}{$R_{s, k}=0.952,(\alpha, \beta)=(0.5,2.5)$} \\
\hline 5 & $(2,2)$ & 0.00239 & 0.00163 & 0.00181 & 1.466 & 1.32 \\
\hline 5 & $(4,4)$ & 0.00124 & 0.00049 & 0.00079 & 2.531 & 1.57 \\
\hline 5 & $(6,6)$ & 0.00070 & 0.00027 & 0.00078 & 2.593 & 0.897 \\
\hline
\end{tabular}

Table 6. MSEs of $R_{s, k}$ estimates and their efficiencies based on SRS, RSS and MRSSO for 5 cycles, $(s, k)=(2$, 4)

\begin{tabular}{|c|c|c|c|c|c|c|}
\hline \multirow{2}{*}{$\boldsymbol{r}$} & \multirow{2}{*}{$\left(m_{x}, m_{y}\right)$} & \multicolumn{3}{|l|}{ MSE } & \multirow{2}{*}{$\begin{array}{l}\mathbf{R E}_{1} \\
\mathbf{R S S} \\
\end{array}$} & \multirow{2}{*}{$\begin{array}{l}\mathbf{R E}_{2} \\
\text { MRSSO } \\
\end{array}$} \\
\hline & & SRS & RSS & MRSSO & & \\
\hline \multicolumn{7}{|c|}{$R_{s, k}=0.752,(\alpha, \beta)=(1.5,2.5)$} \\
\hline 5 & $(3,3)$ & 0.0106 & 0.0057 & 0.0056 & 1.873 & 1.900 \\
\hline 5 & $(5,5)$ & 0.0062 & 0.0027 & 0.0025 & 2.336 & 2.456 \\
\hline 5 & $(7,7)$ & 0.0047 & 0.0013 & 0.0013 & 3.500 & 3.580 \\
\hline \multicolumn{7}{|c|}{$R_{s, k}=0.8,(\alpha, \beta)=(0.5,1)$} \\
\hline 5 & $(3,3)$ & 0.0085 & 0.005 & 0.0058 & 1.706 & 1.478 \\
\hline 5 & $(5,5)$ & 0.0055 & 0.0019 & 0.0023 & 2.82 & 2.389 \\
\hline 5 & $(7,7)$ & 0.0037 & 0.001 & 0.0015 & 3.578 & 2.450 \\
\hline \multicolumn{7}{|c|}{$R_{s, k}=0.91,(\alpha, \boldsymbol{\beta})=(1,3.5)$} \\
\hline 5 & $(3,3)$ & 0.0037 & 0.0022 & 0.0022 & 1.682 & 1.652 \\
\hline 5 & $(5,5)$ & 0.0022 & 0.0009 & 0.0009 & 2.523 & 2.387 \\
\hline 5 & $(7,7)$ & 0.0015 & 0.0005 & 0.0005 & 3.020 & 2.792 \\
\hline \multicolumn{7}{|c|}{$R_{s, k}=0.952,(\alpha, \beta)=(0.5,2.5)$} \\
\hline 5 & $(3,3)$ & 0.0016 & 0.0008 & 0.0027 & 1.952 & 0.607 \\
\hline 5 & $(5,5)$ & 0.0009 & 0.0004 & 0.0004 & 2.300 & 2.629 \\
\hline 5 & $(7,7)$ & 0.0006 & 0.0002 & 0.0002 & 3.050 & 3.211 \\
\hline
\end{tabular}


Table 7. MSEs of $R_{s, k}$ estimates and their efficiencies based on SRS, RSS and MRSS (X has odd set size and $Y$ has even set size) for 5 cycles, $(s, k)=(2,4)$

\begin{tabular}{|c|c|c|c|c|c|c|}
\hline \multirow[t]{2}{*}{$r$} & \multirow{2}{*}{$\left(m_{x}, m_{y}\right)$} & \multicolumn{3}{|l|}{ MSE } & \multirow{2}{*}{$\begin{array}{l}\text { RE }_{1} \\
\text { RSS } \\
\end{array}$} & \multirow{2}{*}{$\begin{array}{l}\mathbf{R E}_{2} \\
\text { MRSS } \\
\end{array}$} \\
\hline & & SRS & RSS & MRSS & & \\
\hline \multicolumn{7}{|c|}{$R_{s, k}=0.752,(\alpha, \beta)=(1.5,2.5)$} \\
\hline 5 & $(3,2)$ & 0.01250 & 0.00784 & 0.00783 & 1.594 & 1.596 \\
\hline 5 & $(3,4)$ & 0.00888 & 0.00490 & 0.00764 & 1.812 & 1.162 \\
\hline \multicolumn{7}{|c|}{$R_{s, k}=0.8,(\alpha, \beta)=(0.5,1)$} \\
\hline 5 & $(3,2)$ & 0.00992 & 0.00681 & 0.00965 & 1.457 & 1.028 \\
\hline 5 & $(3,4)$ & 0.00677 & 0.00391 & 0.00669 & 1.731 & 1.012 \\
\hline \multicolumn{7}{|c|}{$R_{s, k}=0.91,(\alpha, \beta)=(1,3.5)$} \\
\hline 5 & $(3,2)$ & 0.00417 & 0.00274 & 0.00268 & 1.522 & 1.556 \\
\hline 5 & $(3,4)$ & 0.00322 & 0.00172 & 0.00229 & 1.872 & 1.406 \\
\hline \multicolumn{7}{|c|}{$R_{s, k}=0.952,(\alpha, \beta)=(0.5,2.5)$} \\
\hline 5 & $(3,2)$ & 0.00231 & 0.00121 & 0.00102 & 1.909 & 2.265 \\
\hline 5 & $(3,4)$ & 0.0015 & 0.00081 & 0.00085 & 1.852 & 1.765 \\
\hline
\end{tabular}

Table 8. MSEs of $R_{s, k}$ estimates and their efficiencies based on SRS, RSS and MRSS (X has even set size and $Y$ has odd set size) for 5 cycles, $(s, k)=(2,4)$

\begin{tabular}{|c|c|c|c|c|c|c|}
\hline \multirow{2}{*}{$r$} & \multirow{2}{*}{$\left(m_{x}, m_{y}\right)$} & \multicolumn{3}{|l|}{ MSE } & \multirow{2}{*}{$\begin{array}{l}\mathbf{R E}_{1} \\
\text { RSS } \\
\end{array}$} & \multirow{2}{*}{$\begin{array}{l}\mathbf{R E}_{2} \\
\text { MRSS } \\
\end{array}$} \\
\hline & & SRS & RSS & MRSS & & \\
\hline \multicolumn{7}{|c|}{$R_{s, k}=0.752,(\alpha, \beta)=(1.5,2.5)$} \\
\hline 5 & $(2,3)$ & 0.01398 & 0.00889 & 0.01055 & 1.573 & 1.325 \\
\hline 5 & $(4,3)$ & 0.00943 & 0.00458 & 0.00720 & 2.059 & 1.310 \\
\hline \multicolumn{7}{|c|}{$R_{s, k}=0.8,(\alpha, \beta)=(0.5,1)$} \\
\hline 5 & $(2,3)$ & 0.01199 & 0.00823 & 0.01001 & 1.457 & 1.198 \\
\hline 5 & $(4,3)$ & 0.00739 & 0.00401 & 0.00634 & 1.843 & 1.166 \\
\hline \multicolumn{7}{|c|}{$R_{s, k}=0.91,(\alpha, \beta)=(1,3.5)$} \\
\hline 5 & $(2,3)$ & 0.00485 & 0.00314 & 0.00336 & 1.545 & 1.443 \\
\hline 5 & $(4,3)$ & 0.00296 & 0.00157 & 0.00222 & 1.885 & 1.333 \\
\hline \multicolumn{7}{|c|}{$R_{s, k}=0.952,(\alpha, \beta)=(0.5,2.5)$} \\
\hline 5 & $(2,3)$ & 0.00237 & 0.00141 & 0.00131 & 1.681 & 1.809 \\
\hline 5 & $(4,3)$ & 0.00131 & 0.00069 & 0.00125 & 1.899 & 1.048 \\
\hline
\end{tabular}

\section{REAL DATA EXAMPLE}

We provide two data sets for exemplifying purposes and characterize all the details. Data 1 and 2 reported in [36]. The data sets represent the strength for single carbon fibers of lengths $10 \mathrm{~mm}$ and $20 \mathrm{~mm}$ with sample sizes $n=63$ and $m=69$ respectively.

The distance values of the Kolmogorov-Smirnov test for both data are equal to 0.086 and 0.041 with the corresponding $\mathrm{P}$ values as 0.74 and 0.999 respectively.

According to the theoretical results provided in the above sections, we obtain the reliability estimates using the MRSS, RSS and SRS from the mentioned real data sets. Table 9 gives the MSS reliability estimates from the GIE distribution using the proposed sampling designs for selected values of $m_{x}, m_{y}$ for 5 cycles. 
Table 9. MSS reliability estimate of carbon fibres data based on SRS, RSS, MRSSE and MRSSO for 5 cycles

\begin{tabular}{|c|c|c|c|c|c|c|c|}
\hline \multirow{2}{*}{$r$} & \multirow{2}{*}{$\left(m_{x}, m_{y}\right)$} & \multicolumn{3}{|c|}{$(s, k)=(1,3)$} & \multicolumn{3}{|c|}{$(s, k)=(2,4)$} \\
\hline & & SRS & RSS & MRSSE & SRS & RSS & MRSSE \\
\hline 5 & $(2,2)$ & 0.787 & 0.886 & 0.924 & 0.644 & 0.778 & 0.839 \\
\hline 5 & $(4,4)$ & 0.947 & 0.939 & 0.955 & 0.880 & 0.865 & 0.896 \\
\hline 5 & $(6,6)$ & 0.905 & 0.965 & 0.957 & 0.809 & 0.915 & 0.900 \\
\hline$r$ & $\left(\boldsymbol{m}_{x}, \boldsymbol{m}_{y}\right)$ & SRS & RSS & MRSSO & SRS & RSS & MRSSO \\
\hline 5 & $(3,3)$ & \begin{tabular}{|l|}
0.907 \\
\end{tabular} & 0.960 & 0.941 & 0.811 & 0.904 & 0.869 \\
\hline 5 & $(5,5)$ & 0.937 & 0.965 & 0.934 & 0.862 & 0.916 & 0.857 \\
\hline 5 & $(7,7)$ & 0.950 & 0.949 & 0.917 & 0.886 & 0.885 & 0.827 \\
\hline \multirow{2}{*}{$r$} & \multirow{2}{*}{$\left(m_{x}, m_{y}\right)$} & \multicolumn{3}{|c|}{$(s, k)=(1,3)$} & \multicolumn{3}{|c|}{$(s, k)=(2,4)$} \\
\hline & & SRS & RSS & MRSS & SRS & RSS & MRSS \\
\hline 5 & $(3,2)$ & 0.907 & 0.896 & 0.982 & 0.812 & 0.793 & 0.770 \\
\hline 5 & $(3,4)$ & 0.964 & 0.932 & 0.982 & 0.913 & 0.853 & 0.952 \\
\hline$r$ & $\left(m_{x}, m_{y}\right)$ & SRS & RSS & MRSS & SRS & RSS & MRSS \\
\hline 5 & $(2,3)$ & 0.820 & 0.947 & 0.965 & 0.686 & 0.880 & 0.915 \\
\hline 5 & $(4,3)$ & 0.693 & 0.966 & 0.933 & 0.537 & 0.917 & 0.800 \\
\hline
\end{tabular}

\section{CONCLUDING REMARKS}

This article discusses the estimation of the MSS reliability $R_{s, k}$ when $X$ and $Y$ are independent but not identically distributed random variables from the GIE. We consider the reliability estimators viz SRS, RSS and MRSS designs. We inspect the MSS reliability estimators in four distinct cases under MRSS. A numerical investigation is established to measure the attitude of the different estimates in view of the three sampling schemes. According to the outcomes of the study, it is remarked that the MSEs of MSS reliability estimates depending on RSS and MRSS data are smaller than the corresponding in view of SRS data in most of the cases. Also, it can be remarked that RSS is efficient than MRSS compared to SRS in most of the cases (according to the choices of parameter values as well as a number of cycles).

This work implied that the estimates of $R_{s, k}$ when stress and strength data are chosen viz RSS are acceptable than the corresponding under MRSS and SRS. Also, the estimates of $R_{s, k}$ under MRSS are suitable than the estimate of $R_{s, k}$ in case of SRS. Generally, we remarked that the MSS estimates under RSS are convenient than other estimates using MRSS and SRS.

\section{COMPETING INTERESTS}

No conflict of interest was declared by the authors.

\section{ACKNOWLEDGMENTS}

The authors would like to show gratitude to the editor and the anonymous referees, for their effort, valuable and very constructive comments, which have greatly improved the contents of the manuscript.

\section{REFERENCES}

[1] McIntyre, G., "A method for unbiased selective sampling, using ranked sets", Australian Journal of Agricultural Research, 3(4): 385-390, (1952). 
[2] Takahasi, K., Wakimoto, K., "On unbiased estimates of the population mean based on the sample stratified by means of ordering", Annals of the Institute of Statistical Mathematics, 20(1): 1-31, (1968).

[3] Dell, T., Clutter, J., "Ranked set sampling theory with order statistics background", Biometrics, 28(2): 545-555, (1972).

[4] Hassan, A. S., "Modified goodness of fit tests for exponentiated Pareto distribution under selective ranked set sampling", Australian Journal of Basic and Applied Sciences, 6(1): 173-189, (2012).

[5] Hassan, A. S., "Maximum likelihood and Bayes estimators of the unknown parameters for exponentiated exponential distribution using ranked set sampling", International Journal of Engineering Research and Applications, 3(1): 720-725, (2013).

[6] Hassan, A. S., Abd-Elfattah, A. M., Nagy, H. F., "Modified goodness of fit tests for the Weibull distribution based on moving extreme ranked set sampling", In The 48th Annual Conference on Statistics, Computer Science and Operations Research, Faculty of Graduate Studies for Statistical Research, Cairo University, (2013).

[7] Özdemir, Y. A., Ebegil, M., Gökpinar, F., “A test statistic based on ranked set sampling for two normal means", Communications in Statistics-Simulation and Computation, 46(10): 8077-8085, (2017).

[8] Özdemir, Y. A., Ebegil, M., Gökpinar, F., "A test statistic for two normal means with median ranked set sampling", Iranian Journal of Science and Technology, Transactions A: Science, 43(3): 1109-1126, (2019).

[9] Bantan, R., Hassan, A. S., Elsehetry, M., "Zubair Lomax distribution: properties and estimation based on ranked set sampling”, CMC-Comuters, Materials \& Continua, 65(3): 2169-2187, (2020).

[10] Muttlak, H., "Median ranked set sampling”, Journal of Applied Statistical Science, 6: 245-255, (1997).

[11] Birnbaum, Z., "On a use of the Mann-Whitney statistic", Proceedings of the Third Berkeley Symposium on Mathematical Statistics and Probability: Contributions to the Theory of Statistics, Berkeley, California: University of California Press, 1: 13-17, (1956).

[12] Birnbaum, Z., McCarty, R., "A distribution-free upper confidence bound for $\operatorname{Pr}\{Y<X\}$ based on independent samples of $X$ and $Y$ ", The Annals of Mathematical Statistics, 29(2): 558-562, (1958).

[13] Sengupta, S., Mukhuti, S., "Unbiased estimation of $\mathrm{P}(X>Y)$ for exponential populations using order statistics with application in ranked set sampling", Communications in Statistics-Theory and Methods, 37(6): 898-916, (2008).

[14] Muttlak, H. A., Abu-Dayyeh, W., Saleh, M., Al-Sawi, E., "Estimating $\mathrm{P}(Y<X)$ using ranked set sampling in case of the exponential distribution", Communications in Statistics-Theory and Methods, 39(10): 1855-1868, (2010).

[15] Hussian, M. A., "Estimation of stress-strength model for generalized inverted exponential distribution using ranked set sampling", International Journal of Advances in Engineering \& Technology, 6(6): 2354-2362, (2014).

[16] Hassan, A. S., Assar, S., Yahya, M., "Estimation of $\mathrm{R}=\mathrm{P}[Y<X]$ for Burr type XII distribution based on ranked set sampling", International Journal of Basic and Applied Sciences, 3(3): 274-280, (2014). 
[17] Hassan, A. S., Assar, S., Yahya, M., "Estimation of $\mathrm{P}(Y<X)$ for Burr distribution under several modifications for ranked set sampling", Australian Journal of Basic and Applied Sciences, 9(1): 124-140, (2015).

[18] Akgül, F. G., Şenoğlu, B., "Estimation of $\mathrm{P}(X<Y)$ using ranked set sampling for the Weibull distribution", Quality Technology and Quantitative Management, 14(3): 296-309, (2017).

[19] Akgül, F. G., Acıtaş, Ş., Şenoğlu, B., "Inferences on stress-strength reliability based on ranked set sampling data in case of Lindley distribution", Journal of Statistical Computation and Simulation, 88(15): 3018-3032, (2018).

[20] Al-Omari, A. I., Hassan, A. S., Nagy, H. F., "Estimation of the stress-strength reliability for exponentiated Pareto distribution using median and ranked set sampling methods", CMCComputers, Materials \& Continua, 64(2): 835-857, (2020).

[21] Bhattacharyya, G., Johnson, R. A., "Estimation of reliability in a multicomponent stress-strength model”, Journal of the American Statistical Association, 69(348): 966-970, (1974).

[22] Kuo, W., Zuo, M. J., "Optimal Reliability Modeling: Principles and Applications”, John Wiley \& Sons, (2003).

[23] Rezaei, S., Tahmasbi, R., Mahmoodi, M., "Estimation of $\mathrm{P}[Y<X]$ for generalized Pareto distribution", Journal of Statistical Planning and Inference, 140(2): 480-494, (2010).

[24] Hassan, A. S., Basheikh, H. M., "Estimation of reliability in multi-component stress-strength model following exponentiated Pareto distribution", The Egyptian Statistical Journal, Faculty of Graduate Studies for Statistical Research, Cairo University, 56(2): 82-95, (2012).

[25] Hassan, A. S., Basheikh, H. M., "Reliability estimation of stress-strength model with non-identical component strengths: the exponentiated Pareto case", International Journal of Engineering Research and Applications, 2(3): 2774-2781, (2012).

[26] Rao, G. S., "Estimation of reliability in multicomponent stress-strength based on generalized exponential distribution", Revista Colombiana de Estadística, 35(1): 67-76, (2012).

[27] Rao, G. S., Aslam, M., Kundu, D., "Burr-XII distribution parametric estimation and estimation of reliability of multicomponent stress-strength", Communications in Statistics-Theory and Methods, 44(23): 4953-4961, (2015).

[28] Hassan, A. S., Assar, M. S., Yahya, M., "Estimation of reliability in multicomponent stressstrength model following Burr Type XII distribution under selective ranked set sampling", International Journal of Engineering Research and Applications, 5(2): 62-78, (2015).

[29] Hassan, A. S., Nagy, H. F., Muhammed, H. Z., Saad, M. S., "Estimation of multicomponent stressstrength reliability following Weibull distribution based on upper record values", Journal of Taibah University for Science, 14(1): 244-253, (2020).

[30] Abouammoh, A., Alshingiti, A. M., "Reliability estimation of generalized inverted exponential distribution", Journal of Statistical Computation and Simulation, 79(11): 1301-1315, (2009).

[31] Dey, S., Dey, T., "Generalized inverted exponential distribution: Different methods of estimation", American Journal of Mathematical and Management Sciences, 33(3): 194-215, (2014).

[32] Hassan, A. S., Abd-Allah, M., Nagy, H. F., "Bayesian analysis of record statistics based on generalized inverted exponential model", International Journal on Advanced Science, Engineering and Information Technology, 8(2): 323-335, (2018). 
[33] Hassan, A. S., Abd-Allah, M., Nagy, H. F., "Estimation of $\mathrm{P}(Y<X)$ using record values from the generalized inverted exponential distribution", Pakistan Journal of Statistics and Operation Research, 14(3): 645-660, (2018).

[34] Hassan, A. S., Al-Omari, A. I., Nagy, H. F., "Stress-strength reliability for the generalized inverted exponential distribution using MRSS", Iranian Journal of Science and Technology, Transactions A: Science, 45(2): 641-659, (2021).

[35] Krishna, H., Kumar, K., "Reliability estimation in generalized inverted exponential distribution with progressively type II censored sample", Journal of Statistical Computation and Simulation, 83(6): 1007-1019, (2013).

[36] Bader, M., Priest, A., "Statistical aspects of fibre and bundle strength in hybrid composites", Progress in Science and Engineering of Composites: 1129-1136, (1982). 